\title{
Deformation of Second and Third Quantization
}

\author{
Mir Faizal \\ Department of Physics and Astronomy, \\ University of Waterloo, Waterloo, \\ Ontario N2L 3G1, Canada
}

\begin{abstract}
In this paper, we will deform the second and third quantized theories by deforming the canonical commutation relations in such a way that they become consistent with the generalized uncertainty principle. Thus, we will first deform the second quantized commutator and obtain a deformed version of the Wheeler-DeWitt equation. Then we will further deform the third quantized theory by deforming the third quantized canonical commutation relation. This way we will obtain a deformed version of the third quantized theory for the multiverse.
\end{abstract}

\section{Introduction}

In the second quantized models of quantum gravity, all the physical information about the universe can be extracted from the wave function of the universe as it describes the quantum state of the universe [1-2]. This wave function is obtained by taking a sum over all geometries and field configurations which match with a particular field configuration at a spatial section of the spacetime. This approach is called the the Hartle-Hawking no-boundary proposal. In this approach a Wick rotation to Euclidean time makes this integral well defined. This wave function of the universe can also be viewed as a solution of the Wheeler-DeWitt equation [3-4]. The Wheeler-DeWitt equation is a second quantized equation which can be interpreted as the Schroedinger's equation for gravity. However, there is no time in the Wheeler-DeWitt equation because it has to satisfy the time invariance required by general relativity [5].

The wave function of the universe corresponding to various other boundary conditions has been studied. In the Vilenkin proposal the wave function of the universe is obtained by a quantum tunneling transition [6- [7. A baby universe can be created by a quantum fluctuation of the vacuum and this baby universe may eventually jump into an inflationary period and become a parent universe. Here the parent universes are defined to be universes with a large Hubble length and baby universes are defined to be virtual fluctuations of the metric. In this model, there is no reason why only a single baby universe will jump into an inflationary period and become a parent universe. Thus, this model naturally leads to the existence of multiple universes or the multiverse 8. In fact, this model of inflationary in the multiverse is called the chaotic inflationary multiverse. It has been argued in this theory that the total number of distinguishable locally Friedman universes generated by eternal inflation is 
proportional to the exponent of the entropy of inflationary perturbations 9 . The multiverse also appears naturally in the landscape of the string theory [10. This is because there are $10^{500}$ different string theory vacuum states [11, and it is suspected that all these different vacuum states could be real vacuum states of different universes [12.

The Wheeler-DeWitt equation is the Schroedinger's equation for gravity, and just as the creation and annihilation of particles cannot be explained using the first quantized formalism, the creation and annihilation of universes cannot be explained using the second quantized formalism. So, just as we need to go to a second quantized formalism to explain the creation and annihilation of particles, we need to go to a third quantized formalism to explain the creation and annihilation of universes [13-18. In the third quantized formalism the Wheeler-DeWitt equation is viewed as a classical field equation, and a classical action corresponding to it obtained. The addition of interaction terms then accounts for the creation and annihilation of universes. Thus, third quantization is a natural formalism for analysing the multiverse. It may be noted that the third quantization of the Kaluza-Klein theories has been studied 19. In doing so the number density of the universes created from the vacuum were calculated and an attempt was made to statistically explain the compactification.

It may also be noted that recently the deformation of the first quantized theories by a minimum measurable length has been studied. This is because string theory predicts the existence of a minimum length [20]-24]. In fact, even in loop quantum gravity the existence of minimum length turns big bang into a big bounce 25. Strong indication for the existence of a minimum length of the order of the Planck length also occur in black hole physics [26]-27]. However, according to the Heisenberg uncertainty principle there is no limit to the accuracy with which one can measure the momentum or the position of a particle separately. Thus, the minimum observable length is zero. If we want to incorporate the idea of minimum length, then the Heisenberg uncertainty principle has to be modified. This, modified uncertainty principle is called the generalized uncertainty principle [28-41]. In fact, we have to even modify the Heisenberg algebra to make it consistent with this modified uncertainty principle. This modification of the Heisenberg algebra deforms all first quantized Hamiltonians as $H \psi=H_{0} \psi+H_{1} \psi$, where $H_{0}$ is the original Hamiltonian, and $H_{1}=\beta p^{4} / m$ is the term that occurs due to the existence of a minimum length. The second quantization of this deformed first quantized theory has also been studied [42-45. In fact, certain models of inflation motivated by such developments have also been analysed 46-47. In these models the inflationary scalar density perturbations effectively reduces to a minimally coupled massless real scalar field on a fixed curved background spacetime. These scalar perturbations are modified by the existence of a minimum length scale in the theory. Thus, in this work the coordinate representation of the momentum operator for scalar perturbations is modified, and this corresponds to the introduction of higher derivative terms in the action for the minimally coupled massless real scalar field. It is also possible to deform the second quantized commutation relation between any field theory, and this is expected to change the quantum mechanical aspects of such a theory, while leaving the classical equations of motion of such a theory un-deformed . Thus, this deformation is expected to change the kinitic part of the Wheeler-DeWitt equation. In fact, such a deformation of Wheeler-DeWitt equation has already been studied 48. In this paper, we 
will analyse such a deformation of the second quantized commutator by the generalized uncertainty principle. We will also deforming the third quantized commutator by the generalized uncertainty principle.

\section{Deformed Second Quantization}

Various implication of deformed Heisenberg algebra for quantum field theories have been studied 28-41. In this section we will analyse the implication of this deformation for the Wheeler-DeWitt equation. The generalized uncertainty principle is consistent with the following deformed Heisenberg algebra [28]-41]

$$
\left[x^{i}, p_{j}\right]=i \delta_{j}^{i}+i f_{j}^{i}(p),
$$

where $f_{j}^{i}(p)$ is a tensor constructed out of momentum operators. One of the most used representations of $f_{j}^{i}(p)$ is given by

$$
\left[x^{i}, p_{j}\right]=i \delta_{j}^{i}\left[1+\beta p^{k} p_{k}\right]+2 i \beta p^{i} p_{j},
$$

where $\beta$ is a constant. This deformation corresponds to taking the following representation of the momentum operator

$$
p_{i}=-i\left(1+\beta \partial^{j} \partial_{j}\right) \partial_{i} .
$$

Now we can deform the canonical commutation relation in quantum field theory in a similar way. Thus, we can write the commutator of a scalar field theory as

$$
[\phi(x), \pi(y)]=i \delta(x-y)+i f(x-y) .
$$

where $f(x, y)$ are constructed from momentum density conjugate to $\psi$. Now again motivated from the fact that one of the most common forms of the tensor function, $f_{j}^{i}=\delta_{j}^{i} p^{k} p_{k}+2 p^{i} p_{j}$, we write the deformed canonical commutation relation in quantum field theory as

$$
[\phi(x), \pi(y)]=i \delta(x-y)\left[1+\beta \int d z \pi^{2}(z)\right]+2 i \beta \pi(x) \pi(y) .
$$

This corresponds to taking the following representation for $\pi(x)$

$$
\pi(x)=-i\left(1+\beta \int d y d z \frac{\delta}{\delta \phi(y)} \frac{\delta}{\delta \phi(z)} \delta(z-y)\right) \frac{\delta}{\delta \phi(x)} .
$$

Thus, we observe that the deformation of the second quantized canonical commutation relation induces non-locality in the quantum field theory.

Now in the Arnowitt-Deser-Misner $3+1$ decomposition of general relativity, we take the following line element

$$
d s^{2}=g_{\mu \nu}(x) d x^{\mu} d x^{\nu}=\left(-N^{2}+N_{i} N^{i}\right) d t^{2}+2 N_{j} d t d x^{j}+h_{i j} d x^{i} d x^{j} .
$$

where $N_{i}$ the shift function and $N$ is the lapse function. Now we can write the Lagrangian for a universe filled with a cosmological constant $\Lambda$ in terms of the 
the second fundamental form $K_{i j}$, the three dimensional scalar curvature ${ }^{3} R$ and the three dimensional determinant of the metric $\sqrt{{ }^{3} h}$ as,

$$
\mathcal{L}\left[N, N_{i}, h_{i j}\right]=\sqrt{-g} R=\frac{N \sqrt{{ }^{3} h}}{2 \kappa}\left[K_{i j} K^{i j}-K^{2}+\left({ }^{3} R-2 \Lambda\right)\right],
$$

where $K=h^{i j} K_{i j}$ is the trace of the second fundamental form. The conjugate momentum is defined to be

$$
\pi^{i j}=\frac{\delta \mathcal{L}}{\delta\left(\partial_{t} g_{i j}\right)}
$$

The Hamiltonian can be calculated using a Legendre transformation,

$$
\tilde{H}=d x\left[N H+N_{i} H^{i}\right],
$$

where

$$
\begin{aligned}
H & =(2 \kappa) G_{i j k l} \pi^{i j} \pi^{k l}-\frac{\sqrt{{ }^{3} h}}{2 \kappa}\left({ }^{3} R-2 \Lambda\right), \\
H^{i} & =-2 \nabla_{j} \pi^{j i} .
\end{aligned}
$$

Here $G_{i j k l}$ is defined by

$$
G_{i j k l}=\frac{1}{2 \sqrt{h}}\left(h_{i k} h_{j l}+h_{i l} h_{j k}-h_{i j} h_{k l}\right) .
$$

Now the two classical constraints $\mathcal{H}=0$, and $\mathcal{H}^{i}=0$, are obtained through the equation of motion. The wave function of the universe $\psi[h]$, is obtained by promoting the promoting the phase space variable to operators. However, we will now apply a deformed canonical commutation relation,

$$
\begin{aligned}
{\left[h_{i j}(x), \pi^{k l}(y)\right]=} & i \delta(x-y)\left(\delta_{i}^{k} \delta_{j}^{l}+\delta_{i}^{l} \delta_{j}^{k}\right) \\
& \times\left[1+\beta \int d z G_{n m p q} \pi^{m n}(z) \pi^{p q}(z)\right] \\
& +2 i \beta \pi_{i j}(x) \pi^{k l}(y) .
\end{aligned}
$$

It is possible to choose a more general structure for this deformed canonical commutation relation, such that it is consistent with the tensor index symmetry of the equation. However, we will chose this particular form for the deformed canonical commutation relation to simplify further calculations. The momentum operator corresponding to this deformed canonical commutation relation is given by

$$
\pi^{i j}(x)=-i\left(1+2 \beta \int d y d z G_{k l m n} \frac{\delta}{\delta h_{k l}(y)} \frac{\delta}{\delta h_{m n}(z)} \delta(z-y)\right) \frac{\delta}{\delta h_{i j}(x)} .
$$

Thus, we can write the deformed Wheeler-DeWitt equation as

$$
\mathcal{H} \psi[h]=0
$$

where

$$
\begin{aligned}
\mathcal{H}= & -(2 \kappa) G_{i j k l}\left(1+2 \beta \int d y d z G_{n m o p} \frac{\delta}{\delta h_{m n}(y)} \frac{\delta}{\delta h_{o p}(z)} \delta(z-y)\right) \frac{\delta}{\delta h_{i j}(x)} \\
& \times\left(1+2 \beta \int d y d z G_{q r s t} \frac{\delta}{\delta h_{q r}(y)} \frac{\delta}{\delta h_{s t}(z)} \delta(z-y)\right) \frac{\delta}{\delta h_{k l}(x)} \\
& -\frac{\sqrt{3 h}}{2 \kappa}\left({ }^{3} R-2 \Lambda\right) .
\end{aligned}
$$




\section{Third Quantization}

In this section we will analyse a deformation of third quantized canonical commutation relation. In order to analyse the deformation of the third quantization, we need to write the theory in minisuperspace approximation. The FriedmanRobertson-Walker metric for $k=1$ is given by

$$
d s^{2}=-N^{2} d t^{2}+a^{2}(t) d \Omega_{3}^{2},
$$

where $d \Omega_{3}^{2}$ is the usual line element on the three sphere. If the universe is filled with a scalar matter fields $\phi$ and a cosmological constant $\Lambda$, then the Hamiltonian constraint is given by

$$
H=-\frac{\pi_{a}^{2}}{a}+\frac{\pi_{\phi}^{2}}{a^{3}}+V(\phi, a),
$$

where $\pi_{a}$ is the momentum conjugate to $a, \pi_{\phi}$ is the momentum conjugate to $\phi$, and $V(\phi, a)=-a+m^{2} a^{3} \phi^{2}+\Lambda^{3} / 3$. Thus, after applying the deformed canonical commutation relation, the deformed momentum conjugate to $a$ and $\phi$ are given by

$$
\begin{aligned}
& \pi_{a}=-i\left(1+\beta \frac{\partial^{2}}{\partial^{2} a}+\beta \frac{\partial^{2}}{\partial^{2} \phi}\right) \frac{\partial}{\partial a} \\
& \pi_{\phi}=-i\left(1+\beta \frac{\partial^{2}}{\partial^{2} a}+\beta \frac{\partial^{2}}{\partial^{2} \phi}\right) \frac{\partial}{\partial \phi}
\end{aligned}
$$

Now we can write the Wheeler-DeWitt equation as $\mathcal{H} \psi[a, \phi]=0$, where

$$
\begin{aligned}
\mathcal{H}= & \frac{1}{a}\left(\frac{\partial^{2}}{\partial^{2} a}+2 \beta \frac{\partial^{4}}{\partial^{4} a}+2 \beta \frac{\partial^{2}}{\partial^{2} \phi} \frac{\partial^{2}}{\partial^{2} a}\right)+V(\phi, a) \\
& -\frac{1}{a^{3}}\left(\frac{\partial^{2}}{\partial^{2} \phi}+2 \beta \frac{\partial^{2}}{\partial^{2} a} \frac{\partial^{2}}{\partial^{2} \phi}+2 \beta \frac{\partial^{4}}{\partial^{4} \phi}\right) .
\end{aligned}
$$

Thus, we obtained the deformed Wheeler-DeWitt equation in minisuperspace approximation by again deforming the second quantized canonical commutation relation.

We interpret this deformed Wheeler-DeWitt equation in minisuperspace approximation as a classical field equation of a classical field $\psi[\phi, a]$ and view the potential as a spacetime dependent mass term $V(\phi, a)=M^{2}(\phi, a)$. The Lagrangian to be third quantization can now be written as follows,

$$
\begin{aligned}
\mathcal{L}_{\psi}= & \frac{1}{2} \psi[\phi, a]\left[\frac{1}{a}\left(\frac{\partial^{2}}{\partial^{2} a}+2 \beta \frac{\partial^{4}}{\partial^{4} a}+2 \beta \frac{\partial^{2}}{\partial^{2} \phi} \frac{\partial^{2}}{\partial^{2} a}\right)+M^{2}(\phi, a)\right. \\
& \left.-\frac{1}{a^{3}}\left(\frac{\partial^{2}}{\partial^{2} \phi}+2 \beta \frac{\partial^{2}}{\partial^{2} a} \frac{\partial^{2}}{\partial^{2} \phi}+2 \beta \frac{\partial^{4}}{\partial^{4} \phi}\right)\right] \psi[\phi, a] .
\end{aligned}
$$

The variation of this Lagrangian will reproduce the deformed Wheeler-DeWitt equation. Now we can obtain a momentum conjugate to $\psi$ as

$$
P_{\psi}=\frac{\partial \mathcal{L}}{\partial \partial_{a} \psi}
$$


and so we can write the Hamiltonian as

$$
H_{\psi}=P_{\psi} \frac{\partial \psi}{\partial a}-\mathcal{L}_{\psi}
$$

Thus, we have obtained Hamiltonian to be third quantized. Now we impose the deformed third quantized canonical commutation relation

$$
\left[\psi\left(\phi_{1}\right), P_{\psi}\left(\phi_{2}\right)\right]=i \delta\left(\phi_{1}-\phi_{2}\right)\left[1+\beta \int d \phi_{3} P_{\psi}^{2}\left(\phi_{3}\right)\right]+2 i \beta P_{\psi}\left(\phi_{1}\right) P_{\psi}\left(\phi_{2}\right) .
$$

This corresponds to taking the following representation for $P_{\psi}$ in the Hamiltonian $H_{\psi}$,

$$
P_{\psi}(\phi)=-i\left(1+\beta \int d \phi_{1} d \phi_{2} \frac{\delta}{\delta \psi\left(\phi_{1}\right)} \frac{\delta}{\delta \psi\left(\phi_{2}\right)} \delta\left(\phi_{1}-\phi_{2}\right)\right) \frac{\delta}{\delta \psi(\phi)} .
$$

Thus, we can write finally, the deformed third quantized theory as follows,

$$
i \frac{\partial \Psi}{\partial a}=H_{\psi} \Psi,
$$

where $\Psi$ is the wave function of the multiverse. In absence of the matter field $\phi$, this equation becomes,

$$
\left[-\frac{1}{2}\left(\frac{\partial^{2}}{\partial \psi^{2}}+2 \beta \frac{\partial^{4}}{\partial \psi^{4}}\right)+\frac{\tilde{\omega}^{2}(a)}{2}\right] \Psi(\psi, a)=i \frac{\partial \Psi(\psi, a)}{\partial a} .
$$

This corresponds appears like a time dependent equation for a deformed Harmonic oscillator with time dependent frequency. We can also define a quantity analogous to energy for this case, such that $H_{\psi} \Psi=E \Psi$. Now wave function is known that the wave function for the un-deformed case can be written as [53]-54] $\mid \Psi_{n_{1}, n_{2}}(x, y)>$, then the deformation corresponds to the following by treating the

$$
\Delta \Psi_{n_{1}, n_{2}}(\psi, a)=\sum_{\left.\left\{m_{1}, m_{2}\right\} \neq\left\{n_{1}, n_{2}\right)\right\}} \frac{M_{m_{1}, m_{2}, n_{1}, n_{2}}(\psi, a) \Psi_{m_{1}, m_{2}}(\psi, a)}{E_{n_{1}, n_{2}}-E_{m_{1}, m_{2}}},
$$

where

$$
M_{m_{1}, m_{2}, n_{1}, n_{2}}(\psi, a)=<\Psi_{m_{1}, m_{2}}(\psi, a)\left|2 \beta \frac{\partial^{4}}{\partial \psi^{4}}\right| \Psi_{n_{1}, n_{2}}(\psi, a)>.
$$

It is known that the conventional third quantization of the Wheeler-DeWitt equation lead to the vanishing of the cosmological constant [55]-56]. However, there as the third quantized wave function also changed by this deformation, it is possible that by using the deformed third quantization of the WheelerDeWitt equation, a small but finite cosmological constant might be obtained. This is what would be expected from experiments, as it has been found that our universe is in a state of accelerated expansion [57-62].

The Wheeler-DeWitt equation describes the evolution of the wave function in the second quantized formalism. To deal with the creation and annihilation of universes, we used a third quantized formalism. In the third quantized formalism, Wheeler-DeWitt equation was viewed as classical field equation of a 
classical field. Thus, an action for this classical field was constructed such that its equation of motion coincides with the second quantized Wheeler-DeWitt equation. This Lagrangian was used for calculate the momentum conjugate to the field variables. After obtaining the momentum conjugate to the field variables, we constructed a deformed third quantized theory by deforming the third quantized canonical commutation relation. It may be noted that in the third quantized theory without matter fields, $\psi$ acts like the space coordinate and $a$ acts like the time coordinate. The deformed third quantized equation obtained in this case is similar to a quantum mechanical equation quantized using generalized uncertainty principle. The existence of a minimum length can be inferred from this generalized uncertainty principle [28-41]. Now by using the general arguments used in inferring the existence of a minimum length, we can show that the there exists a minimum value for the wave function in this deformed third quantized theory. This minimum value for the wave function in the deformed third quantized theory is given by $\psi_{\min } \sim \sqrt{3 \beta} \neq 0$, as $\beta \neq 0$. So, the wave function of the universe cannot become zero in this deformed third quantized theory. In other words the only way for the wave function of the universe to vanish is that the third quantized deformation parameter is set equal to zero, and this will reduce the deformed third quantized theory to the un-deformed third quantized theory. Alternatively, the non-vanishing of the wave function of the universe implies the deformation of the third quantized theory.

\section{Conclusion}

The Wheeler-DeWitt equation is the Schroedinger's equation for a single universe. In the paper we obtained a deformed Wheeler-DeWitt equation by deforming the second quantized canonical commutation relation. The WheelerDeWitt equation has to be third quantized to obtain the wave function of the multiverse. This was done by first considering the Wheeler-DeWitt equation as a classical field equation and then writing a Lagrangian corresponding to it. This Lagrangian was used for obtaining a momentum conjugate to field variables. After that the theory was third quantized by imposing canonical commutation relation. We also deformed these third quantized canonical commutation relation. Thus, we analysed a deformation of both the second and third quantized canonical commutation relations. It may be noted that the generalized uncertainty principle has been known to deform the first quantized theories. This is what motivated a similar deformation of the second and third quantized canonical commutation relations. We also argued using this logic that the wave function of the universe cannot vanish for a deformed third quantized theory, without reducing this theory to the un-deformed theory. It will be interesting to analyse further the implications of such a deformed quantization of field theories.

The quantum fluctuations in spacetime at Planck scale will give rise to fuzzy structure of spacetime called the spacetime foam 63] 64. In this model the spacetime is populated with virtual Lorentzian and Euclidean wormholes. The Lorentzian wormholes are solutions to the Einstein's equations with at least two asymptotically flat regions. They thus connecting two separate parts either of the same universe or of two different universes. The Euclidean wormholes are Euclidean sectors of a Friedman spacetime. The quantum state of these 
Euclidean wormholes is obtained from a Wick rotation of oscillatory universes. The fluctuation of spacetime at Planck scale can also lead to the formation of virtual black holes. Third quantization is a natural formalism for analysing different models of spacetime foam. In fact, virtual black holes have been studied using third quantization [65. It will be interesting to analyse the effect that this deformed quantization can have on different models of spacetime foam. In fact, the conventional third quantization of the Wheeler-DeWitt equation lead to the vanishing of the cosmological constant [55]-56. It will be interesting to study what happens to the cosmological constant problem using this deformed third quantization. It has already been observed in this paper that the deformation of third quantization corresponding to generalized uncertainty principle, will change the wave function of the universe. Hence, it is hoped that this deformation can lead to the existence of a small but finite cosmological constant.

\section{References}

[1] J. B. Hartle and S. W. Hawking, Phys. Rev. D 28, 2960 (1983)

[2] S. W. Hawking, T. Hertog and H. Reall, Phys. Rev. D 62, 043501 (2000)

[3] B. S. DeWitt, Phys. Rev. 160, 1113 (1967)

[4] J. A. Wheeler, Ann. Phys. 2, 604 (1957)

[5] S. W. Hawking, Pontif. Acad. Sci. Scrivaria 48, 563 (1982)

[6] A. Vilenkin, Phys. Rev. D 33, 3560 (1986)

[7] A. Vilenkin, Phys. Lett. B 117, 25 (1982)

[8] A. Linde, Mod. Phys. Lett A 1, 81 (1986)

[9] A. Linde and V. Vanchurin, Phys. Rev. D81, 083525 (2010)

[10] S. K. Ashok and M. R. Douglas, JHEP 01, 060 (2004)

[11] F. Denef and M. R. Douglas, JHEP 0405, 072 (2004)

[12] L. M. Houghton and R. Holman, JCAP 0902, 006 (2009)

[13] A. Strominger, Nucl. Phy. B 321, 481 (1989)

[14] M. Faizal, Class. Quant. Grav. 29, 215009 (2012)

[15] M. Faizal, J. Exp. Theor. Phys. 114, 400 (2012)

[16] M. Faizal, Mod. Phys. Lett. A27, 1250007 (2012)

[17] M . Faizal, Phys. Lett. B727, 536 (2013)

[18] Y. Ohkuwa and Y. Ezawa, Class. Quant. Grav. 29, 215004 (2012)

[19] Y. Ohkuwa, Int. J. Mod. Phys. A 13, 4091 (1998)

[20] D. Amati, M. Ciafaloni and G. Veneziano, Phys. Lett. B 216, 41 (1989) 
[21] A. Kempf, G. Mangano, and R. B. Mann, Phys. Rev. D 52, 1108 (1995)

[22] L. N. Chang, D. Minic, N. Okamura, and T. Takeuchi, Phys.Rev. D65, $125027(2002)$

[23] L. N. Chang, D. Minic, N. Okamura, and T. Takeuchi, Phys. Rev. D65, $125028(2002)$

[24] S. Benczik, L. N. Chang, D. Minic, N. Okamura, S. Rayyan, and T. Takeuchi, Phys. Rev. D66, 026003 (2002)

[25] P. Dzierzak, J. Jezierski, P. Malkiewicz, and W. Piechocki, Acta Phys. Polon. B41, 717 (2010)

[26] M. Maggiore, Phys. Lett. B304, 65 (1993)

[27] M. I. Park, Phys. Lett. B659, 698 (2008)

[28] D. Amati, M. Ciafaloni, and G. Veneziano, Phys. Lett. B 216, 41 (1989)

[29] M. Maggiore, Phys. Lett. B 304, 65 (1993)

[30] M. Maggiore, Phys. Rev. D 49, 5182 (1994)

[31] M. Maggiore, Phys. Lett. B 319, 83 (1993)

[32] L. J. Garay, Int. J. Mod. Phys. A 10, 145 (1995)

[33] F. Scardigli, Phys. Lett. B 452, 39 (1999)

[34] C. Bambi, F. R. Urban, Class. Quantum Grav. 25, 095006 (2008)

[35] K. Nozari, Phys. Lett. B. 629, 41 (2005)

[36] K. Nozari, T. Azizi, Gen. Relativ. Gravit. 38, 735 (2006)

[37] P. Pedram, Int. J. Mod. Phys. D 19, 2003 (2010)

[38] A. Kempf, J. Phys. A 30, 2093 (1997)

[39] F. Brau, J. Phys. A 32, 7691 (1999)

[40] K. Nozari, and B. Fazlpour, Chaos, Solitons and Fractals, 34, 224 (2007)

[41] S. Das, and E. C. Vagenas, Phys. Rev. Lett. 101, 221301 (2008)

[42] M. Kober, Phys. Rev. D82, 085017 (2010)

[43] M. Kober, Int. J. Mod. Phys. A 27, 1250106 (2012)

[44] V. Husain, D. Kothawala and S. S. Seahra, Phys. Rev. D 87, 025014 (2013)

[45] M. Kober, Int. J. Mod. Phys. A 26, 4251 (2011)

[46] A. Ashoorioon, A. Kempf and R. B. Mann, Phys. Rev. D 71, 023503 (2005)

[47] A. Kempf, Phys. Rev. D63, 083514 (2001)

[48] M. Faizal, Int. J. Mod. Phys. A29, 1450106 (2014) 
[49] L. S. Brown, Phys. Rev. Lett. 18, 510 (1967)

[50] H. R. Lewis, Phys. Rev. Lett. 18, 510 (1967)

[51] H. R. Lewis, J. Math. Phys. 9, 1976 (1968)

[52] H. J. Korsch, Phys. Lett. A 109, 313 (1985)

[53] D. Y. Song, Phys. Rev. A 62, 014103 (2000)

[54] D. Y. Song, Phys. Rev. Lett. 85, 1141 (2000)

[55] S. Coleman, Nucl. Phys. B 310, 643 (1988)

[56] S. Cokman, Nucl. Phys. B 307, 867 (1988)

[57] A.G. Riess et al., Astron. J. 116, 1009 (1998)

[58] S. Perlmutter et al., Nature 391, 51 (1998)

[59] A. G. Riess et al., Astron. J. 118, 2668 (1999)

[60] S. Perlmutter et al., Astrophys. J. 517, 565 (1999)

[61] A. G. Riess et al., Astrophys. J. 560, 49 (2001)

[62] J. L. Tonry et al., Astrophys. J. 594, 1 (2003)

[63] S. W. Hawking, Phys. Rev. D 37, 904 (1988)

[64] S. W. Hawking and D. N. Page, Phys. Rev. D 42, 2655 (1990)

[65] M. Faizal, JETP. 114, 400 (2012) 